\title{
Does the Risk Assessment and Prediction Tool Predict Discharge Disposition After Joint Replacement?
}

\author{
Viktor J. Hansen MD, Kirill Gromov MD, PhD, \\ Lauren M. Lebrun MHA, Harry E. Rubash MD, \\ Henrik Malchau MD, PhD, Andrew A. Freiberg MD
}

Published online: 9 August 2014

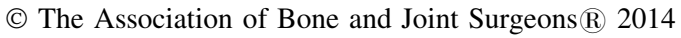

\begin{abstract}
Background Payers of health services and policymakers place a major focus on cost containment in health care. Studies have shown that early planning of discharge is essential in reducing length of stay and achieving financial benefit; tools that can help predict discharge disposition would therefore be of use. The Risk Assessment and Prediction Tool (RAPT) is a preoperative survey constructed to predict discharge disposition after total joint arthroplasty (TJA). The RAPT was developed and tested on a population of Australian patients undergoing joint replacement,
\end{abstract}

Each author certifies that he or she, or a member of his or her immediate family, has no funding or commercial associations (eg, consultancies, stock ownership, equity interest, patent/licensing arrangements, etc) that might pose a conflict of interest in connection with the submitted article.

All ICMJE Conflict of Interest Forms for authors and Clinical Orthopaedics and Related Research ${ }^{\mathbb{R}}$ editors and board members are on file with the publication and can be viewed on request.

Each author certifies that his or her institution approved the human protocol for this investigation, that all investigations were conducted in conformity with ethical principles of research, and that informed consent for participation in the study was obtained.

This work was performed at the Harris Orthopaedic Laboratory, Massachusetts General Hospital, Boston, MA, USA.

V. J. Hansen, K. Gromov, H. Malchau

Harris Orthopaedic Laboratory, Massachusetts General Hospital, Boston, MA, USA

L. M. Lebrun, H. E. Rubash, H. Malchau, A. A. Freiberg ( $\square)$ Department of Orthopaedic Surgery, Massachusetts General Hospital, 55 Fruit Street, Yawkey Suite 3B, Boston, MA 02114, USA

e-mail: afreiberg@mgh.harvard.edu; afreiberg@partners.org

K. Gromov

Department of Orthopaedic Surgery, Copenhagen University Hospital, Hvidovre, Copenhagen, Denmark but its validity in other populations is unknown. A low RAPT score is reported to indicate a high risk of needing any form of inpatient rehabilitation after TJA, including short-term nursing facilities.

Questions/purposes This study attempts (1) to assess predictive accuracy of the RAPT on US patients undergoing total hip and knee arthroplasty (THA/TKA); and (2) to determine predictive accuracy of each individual score (1-12).

Methods Between June 2006 and December 2011, RAPT scores of 3213 patients (1449 THAs; 1764 TKAs) were prospectively captured during the preoperative clinical visit. Scores were stored along with other clinical data, including discharge disposition, in a dedicated database on a secure server. The database was queried by the nursing case manager to retrieve the RAPT scores of all patients captured during this time period. Binary logistic regression was used to analyze the scores and determine predictive accuracy.

Results Overall predictive accuracy was 78\%. RAPT scores $<6$ and $>10$ (of 12) predicted with $>90 \%$ accuracy discharge to inpatient rehabilitation and home, respectively. Predictive accuracy was lowest for scores between 7 and 10 at $65.2 \%$ and almost $50 \%$ of patients received scores in this range. Based on our findings, the risk categories in our populations should be high risk $<7$, intermediate risk 7 to 10 , and low risk $>10$.

Conclusions The RAPT accurately predicted discharge disposition for high- and low-risk patients in our cohort. Based on our data, intermediate-risk patients should be defined as those with scores of 7 to 10 . Predictive accuracy for these patients could potentially be improved through the identification and addition of other factors correlated to discharge disposition. The RAPT allows for identification of patients who are likely to be discharged home or to 
rehabilitation, which may facilitate preoperative planning of postoperative care. Additionally, it identifies intermediate-risk patients and could be used to implement targeted interventions to facilitate discharge home in this group of patients.

Level of Evidence Level III, diagnostic study. See the Guidelines for Authors for a complete description of levels of evidence.

\section{Introduction}

Total joint arthroplasties (TJAs) are some of the most commonly performed elective orthopaedic procedures with more than 1,000,000 THAs and TKAs estimated to be performed annually in the United States [4]. Although they are effective treatments for osteoarthritis [6-9, 12], the costs associated with these procedures are substantial $[1,2$, 10]. In recent years, payers of health services and policymakers have placed a major focus on cost containment in health care. Decreasing length of stay has been a key element of this emphasis. In addition, surgeons, patients, and policymakers have noted the importance of planning for discharge before the performance of elective procedures.

The Risk Assessment and Prediction Tool (RAPT) is a preoperative survey constructed to predict discharge disposition after TJA [11]. It consists of six questions and generates a score from 1 to 12 with a low score indicating a higher risk for needing inpatient rehabilitation after TJA, including short-term nursing facilities (SNFs). The tool was developed and validated on data from an Australian cohort of 650 patients undergoing TJA. The cohort was split into two groups; data from the first 530 patients were used to develop the tool and data from the next 120 patients were used for validation. The reported overall predictive accuracy was $75 \%$ [11].

The purposes of this study were to (1) assess the predictive accuracy of the RAPT on a larger cohort of US patients undergoing THA and TKA; and (2) determine the predictive accuracy of each of the individual scores (1-12).

\section{Patients and Methods}

The six items included in the RAPT are age, sex, preoperative walking distance, use of a gait aid, community supports, and the presence of a caregiver on return home. The questions in the score are weighted differently and the number of points assigned to each question depends on their relative correlation to discharge disposition. A score between 1 and 12 is generated based on a patient's answers to the questions (Table 1). Patients with scores $<6$ and $>9$
Table 1. Items included in the Risk Assessment and Prediction Tool (RAPT) and the score for each item

\begin{tabular}{lll}
\hline Item & Value & Score \\
\hline Age group (years) & $50-65$ & 2 \\
& $66-75$ & 1 \\
\multirow{3}{*}{ Sex } & $>75$ & 0 \\
& Male & 2 \\
Walking distance & Female & 1 \\
& Two blocks or more & 2 \\
Use of gait aid & 1-2 blocks & 1 \\
& Housebound & 0 \\
& None & 2 \\
Use of community supports & Single-point stick & 1 \\
& Crutches/frame & 0 \\
Caregiver at home & None or one per week & 1 \\
& Two or more per week & 0 \\
& Yes & 3 \\
& No & 0 \\
\hline
\end{tabular}

Table 2. Patient demographics and discharge disposition

\begin{tabular}{cllll}
\hline Procedure & $\begin{array}{l}\text { Number of } \\
\text { patients }\end{array}$ & $\begin{array}{l}\text { Age } \pm \text { SD } \\
\text { (years) }\end{array}$ & $\begin{array}{l}\text { Sex } \\
\text { (male) }\end{array}$ & $\begin{array}{l}\text { Discharge } \\
\text { disposition (home) }\end{array}$ \\
\hline $\begin{array}{c}\text { Primary } \\
\text { THA }\end{array}$ & 1449 & $66.0 \pm 13.3$ & $49.7 \%$ & $59.0 \%$ \\
$\begin{array}{c}\text { Primary } \\
\text { TKA }\end{array}$ & 1764 & $67.3 \pm 10.0$ & $41.0 \%$ & $51.2 \%$ \\
\hline
\end{tabular}

have a high and low risk of needing any form of inpatient rehabilitation, respectively, whereas patients with scores between 6 and 9 are considered to have an intermediate risk.

As of June 2006, the RAPT was made available to patients scheduled for TJA at Massachusetts General Hospital (MGH). Scores were prospectively captured preoperatively by the nursing case manager and stored in a dedicated database on a secure server along with various other procedural data including discharge disposition. The number of scores that could be captured was limited by the caseload of the nursing case manager. RAPT scores captured in 2008 and 2009 were lost during transition to a new computer system and were therefore not available for analysis. The database was queried by the nursing case manager to retrieve the RAPT scores of all patients captured between June 2006 and December 2011. RAPT scores for 3213 patients undergoing TJA (1449 THAs; 1764 TKAs) were available in the database during the study period (Table 2).

Binary logistic regression analysis was used to assess the predictive accuracy of the RAPT and calculate predicted probabilities to discharge home for each of the 
Table 3. Predictive accuracy of the RAPT for all patients and for specific risk groups

\begin{tabular}{lrrrr}
\hline Cohort & Overall & Home & Rehabilitation & p value \\
\hline All patients & $78.3 \%$ & $82.4 \%$ & $73.4 \%$ & $<0.0001$ \\
$\quad$ THA & $79.6 \%$ & $91.1 \%$ & $63.0 \%$ & $<0.0001$ \\
TKA & $77.4 \%$ & $83.3 \%$ & $71.3 \%$ & $<0.0001$ \\
Original high risk (0-5) & $92.8 \%$ & $0.0 \%$ & $100.0 \%$ & $<0.0001$ \\
High risk (0-6) & $89.4 \%$ & $0.0 \%$ & $100.0 \%$ & $<0.0001$ \\
$\quad$ Original intermediate & $66.0 \%$ & $44.0 \%$ & $79.8 \%$ & $<0.0001$ \\
$\quad$ risk (6-9) & & & & \\
Intermediate risk (7-10) & $65.2 \%$ & $100.0 \%$ & $0.0 \%$ & $<0.0001$ \\
$\quad$ Original low risk & $84.4 \%$ & $100.0 \%$ & $0.0 \%$ & $<0.0001$ \\
$\quad(10-12)$ & & & & \\
Low risk (11-12) & $89.5 \%$ & $100.0 \%$ & $0.0 \%$ & $<0.0001$ \\
\hline
\end{tabular}

RAPT scores (1-12). RAPT score and discharge disposition were used as the independent and dependent variables, respectively. Predictive accuracy was assessed for all patients combined as well groupwise for high-risk (score 0-5), intermediate-risk (score 6-9), and low-risk patients (score 10-12).

Discharge disposition in this study was divided into two groups: home and rehabilitation, with the latter being defined as any inpatient rehabilitation facility or SNF. The decision on discharge disposition was clinically based and made by the surgeon based on input from physical therapy and the case manager and patient's preference. Insurance status did not have an influence on whether the patient received inpatient or outpatient rehabilitation. All statistical analyses were performed using PASW Statistics 17 (SPSS Inc, Chicago, IL, USA).

\section{Results}

The overall predictive accuracy of the RAPT (all scores) was $78 \%$ in our cohort of patients undergoing TJA. Predictive accuracy was $80 \%$ and $77 \%$ for patients undergoing THA and TKA, respectively. The results of the model show that the RAPT is more successful in accurately predicting patients who are discharged home than those discharged to an inpatient rehabilitation facility (Table 3). Fifty-five percent of patients with RAPT were discharged home after TJA and 45\% were discharged to any type of inpatient rehabilitation facility. Patients after THA were discharged home $59 \%$ of the time, whereas patients who underwent TKA were discharged home in $51 \%$ of cases (Table 2).

RAPT scores $<7$ and $>10$ had the highest predictive accuracy at almost $90 \%$ for both groups, whereas scores between 7 and 10 had the lowest predictive accuracy at
65\% (Table 3). Scores between 7 and 10 all had predictive accuracies less than $75 \%$, whereas scores $<7$ and $>10$ had predictive accuracies higher than $83 \%$. The cohort of patients with scores between 7 and 10 consisted of 1477 of 3213 patients, making up $46 \%$ of the entire study cohort (Table 4; Fig. 1). Having a RAPT score of 6 equals having a $16 \%$ probability of going home and hence a score of $84 \%$ is predictive accuracy of not going home. RAPT scores $<6$ showed a remarkably high accuracy in predicting discharge disposition. All scores $<6$ had at least $90 \%$ predictive accuracy for both patients undergoing THA and those undergoing TKA. The same was true for having a RAPT score of 12 , which predicts discharge home in $96 \%$ of THAs and $94 \%$ of TKAs. By contrast, a score of 10 translates into a $73 \%$ chance of going home and therefore a probability of $27 \%$ of not going home.

\section{Discussion}

As part of an institutional effort to reduce cost and length of stay, we began using RAPT in 2006. The RAPT tool, originally described by Australian researchers [11], is designed to predict discharge disposition of patients undergoing TJA, thus allowing the surgeon and the entire healthcare staff to optimize care according to the patient's specific needs. RAPT uses six criteria with categorical answers for each item with possible total scores of 1 to 12 . We therefore sought to analyze the following: (1) the predictive accuracy of the RAPT on a cohort of US patients undergoing THA and TKA; and (2) the predictive accuracy of each of the individual scores.

There are several limitations to our study. First, patient characteristics and organizational aspects vary greatly not only between countries [3], but also hospitals [15], making our data difficult to extend to other medical institutions. This is illustrated by the significantly different distributions in RAPT scores among patients found in our study compared with those reported by Dauty et al. [5] and Tan et al. [14]. In addition, an intermediate RAPT score might result in very different discharge dispositions in different care systems. Second, our institution does not have strict discharge criteria, therefore increasing the variability in LOS between patients. Third, although equal access to free health care in some European countries makes comparison of discharge disposition easier, studies from the United States show that type of insurance coverage plays a role in patient discharge disposition and postoperative complications [4, 13], thus acting as a potential confounder, because we did not include insurance status in our analysis. It is plausible that patients with medical insurance that covers prolonged stay at a rehabilitation facility will opt to do so despite the surgeon recommending discharge straight to 
Table 4. Distribution of RAPT scores, predicted probabilities, and predictive accuracies*

\begin{tabular}{|c|c|c|c|c|c|c|c|}
\hline $\begin{array}{l}\text { RAPT } \\
\text { score }\end{array}$ & $\begin{array}{c}\text { Number of } \\
\text { patients }\end{array}$ & PP overall & $\begin{array}{l}\text { PA } \\
\text { overall }\end{array}$ & $\begin{array}{l}\text { PP } \\
\text { THA }\end{array}$ & $\begin{array}{l}\text { PA } \\
\text { THA }\end{array}$ & $\begin{array}{l}\text { PP } \\
\text { TKA }\end{array}$ & $\begin{array}{l}\text { PA } \\
\text { TKA }\end{array}$ \\
\hline 1 & $21(0.7 \%)$ & $4.8 \%$ & $95.2 \%$ & $7.1 \%$ & $92.9 \%$ & $0.0 \%$ & $100.0 \%$ \\
\hline 2 & $42(1.3 \%)$ & $2.4 \%$ & $97.6 \%$ & $4.2 \%$ & $95.8 \%$ & $0.0 \%$ & $100.0 \%$ \\
\hline 3 & $69(2.1 \%)$ & $5.8 \%$ & $94.2 \%$ & $5.9 \%$ & $94.1 \%$ & $5.7 \%$ & $94.3 \%$ \\
\hline 4 & $130(4.0 \%)$ & $7.7 \%$ & $92.3 \%$ & $7.0 \%$ & $93.0 \%$ & $8.2 \%$ & $91.8 \%$ \\
\hline 5 & $211(6.6 \%)$ & $8.5 \%$ & $91.5 \%$ & $9.1 \%$ & $90.9 \%$ & $8.1 \%$ & $91.9 \%$ \\
\hline 6 & $266(8.3 \%)$ & $16.5 \%$ & $83.5 \%$ & $18.9 \%$ & $81.1 \%$ & $14.8 \%$ & $85.2 \%$ \\
\hline 7 & $296(9.2 \%)$ & $28.4 \%$ & $71.6 \%$ & $31.7 \%$ & $68.3 \%$ & $26.0 \%$ & $74.0 \%$ \\
\hline 8 & $343(10.7 \%)$ & $43.1 \%$ & $56.9 \%$ & $50.9 \%$ & $50.9 \%$ & $36.1 \%$ & $63.9 \%$ \\
\hline 9 & $376(11.7 \%)$ & $57.7 \%$ & $57.7 \%$ & $63.4 \%$ & $63.4 \%$ & $53.5 \%$ & $53.5 \%$ \\
\hline 10 & $462(14.4 \%)$ & $73.4 \%$ & $73.4 \%$ & $80.0 \%$ & $80.0 \%$ & $68.8 \%$ & $68.8 \%$ \\
\hline 11 & $561(17.5 \%)$ & $85.4 \%$ & $85.4 \%$ & $86.7 \%$ & $86.7 \%$ & $84.3 \%$ & $84.3 \%$ \\
\hline 12 & $436(13.6 \%)$ & $94.7 \%$ & $94.7 \%$ & $95.7 \%$ & $95.7 \%$ & $93.5 \%$ & $93.5 \%$ \\
\hline \multirow[t]{2}{*}{ Total } & 3213 & & & & & & \\
\hline & $(100 \%)$ & $54.7 \%$ & $78.3 \%$ & $58.9 \%$ & $79.6 \%$ & $51.2 \%$ & $77.4 \%$ \\
\hline
\end{tabular}

* The four scores with the lowest predictive accuracy, representing the intermediate-risk scores, are outlined by a rectangle; PP = predicted probability; PA = predictive accuracy.

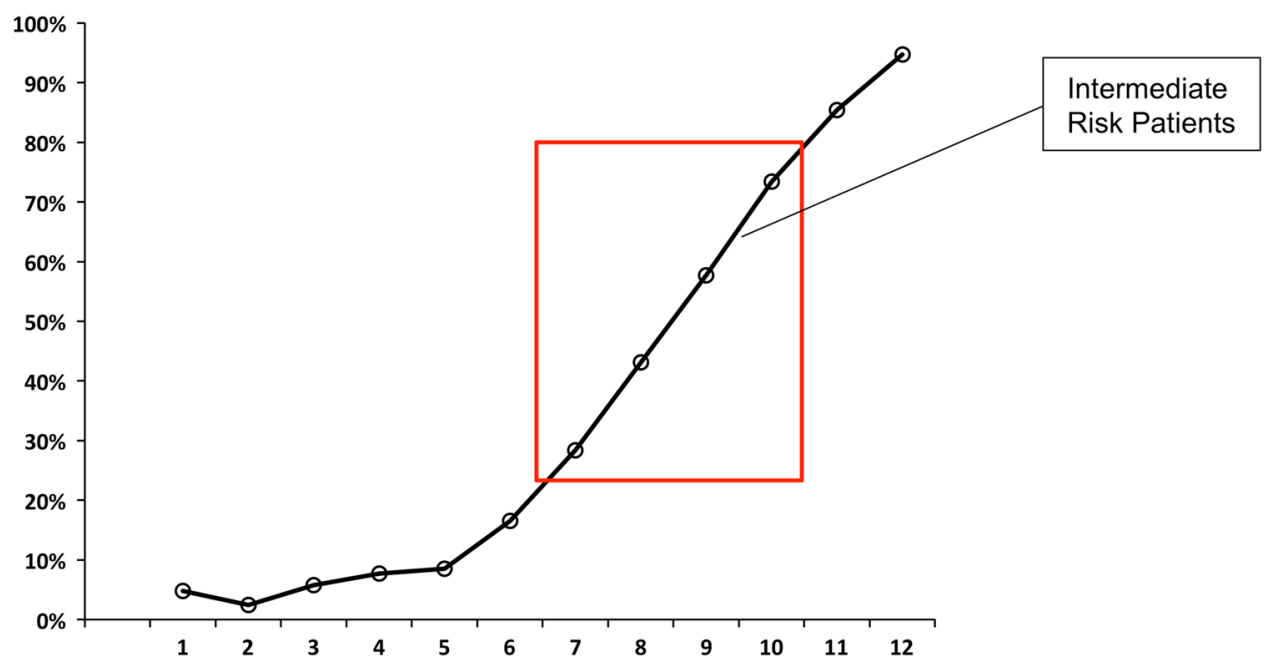

Fig. 1 Predictive accuracy of the RAPT is displayed as a plot of each score (1-12) versus the proportion of patients with that score who were discharged home. The scores of intermediate-risk patients in our cohort (score 7-10) are represented by the red rectangle.

home. Fourth, we did not differentiate among discharge to home with no assistance, discharge to home with home health assistance, discharge to an acute inpatient rehabilitation facility, or discharge to a SNF. Most importantly, we found that half of our patients received intermediate scores, which accurately predicted outcome in only $65 \%$ of cases.

We first assessed the predictive accuracy of the RAPT in a large cohort of patients undergoing THA and TKA. The overall predictive accuracy was comparable to the one reported by Oldmeadow et al. [11]. The original investigation of RAPT found that inclusion of medical comorbidities, perhaps surprisingly, provided no additional benefit to predictive accuracy. Thus, we did not attempt to incorporate scoring of overall medical condition such as the Charlson Index in our study.

We next evaluated the results for each score. We noted that both very high and very low scores were highly predictive of discharge destination but that intermediate scores were less so. Although the overall predictive accuracy of the RAPT in our study was similar to the one reported by Oldmeadow et al. [11], the scores of the intermediate-risk patients differed. The RAPT classifies scores of 6 to 9 as intermediate risk, whereas in our study, the scores with the lowest predictive accuracy were 7 to 10 . These findings 
suggest that in our cohort of patients, the risk categories should be defined as follows: high risk $<7$, intermediate risk 7 to 10, and low risk $>10$. Patients with intermediaterisk scores comprised $46 \%$ of our cohort and their discharge disposition could be accurately predicted in only $65 \%$ of cases. Compared with a pretest probability for any patient discharging home of $55 \%$, this represents a mere 10 percentage point increase in predictive capacity. It is difficult to speculate as to why the RAPT scores with the lowest predictive accuracy differ between the two studies. Our results indicate that a patient in our US cohort needs a higher score than an Australian patient to not have a high risk of discharging to an inpatient rehabilitation facility (7 versus 6). Furthermore, our results indicate that a patient in our cohort needs a higher score to have a high chance of discharging home (10 versus 11). Perhaps these results reflect the difference in practice between the two countries in terms of use of inpatient rehabilitation facilities. This could be related to a number of factors such as patient preference, surgeon preference, and availability of inpatient rehabilitation facilities.

As part of an integrated clinical care pathway, preoperative scoring of likelihood of discharge is an attractive prospect, because it allows caregivers to focus on patients who require the most assistance with obtaining safe and appropriate postoperative care. The use of the RAPT in terms of preoperatively planning postoperative care is limited by the fact that almost $50 \%$ of the patients fall within the intermediate-risk category. This does, however, allow for identification of patients for targeted interventions that could potentially increase the likelihood of these patients being discharged home. Studies investigating additional factors correlated to discharge disposition such as patient-reported outcome measures, body mass index, and American Society of Anesthesiologists score are currently being performed at MGH and could potentially improve the predictive accuracy of the RAPT for intermediate-risk patients. Further studies assessing the predictive accuracy of preoperative scoring systems such as RAPT at other institutions are warranted as well as studies assessing the effect of using RAPT to identify patients for targeted interventions in terms of length of stay, discharge disposition, clinical outcomes, and financial impact.

Acknowledgments We thank Pamela Tobichuk and Robert Dorman for their assistance in preparing the manuscript.

\section{References}

1. Bozic KJ, Katz P, Cisternas M, Ono L, Ries MD, Showstack J. Hospital resource utilization for primary and revision total hip arthroplasty. J Bone Joint Surg Am. 2005;87:570-576.

2. Bozic KJ, Stacey B, Berger A, Sadosky A, Oster G. Resource utilization and costs before and after total joint arthroplasty. BMC Health Serv Res. 2012;12:73.

3. Bozic KJ, Wagie A, Naessens JM, Berry DJ, Rubash HE. Predictors of discharge to an inpatient extended care facility after total hip or knee arthroplasty. J Arthroplasty. 2006;21:151-156.

4. Centers for Disease Control and Prevention. National Hospital Discharge Survey: 2010. Available at: www.cdc.gov/nchs/data/ nhds/4procedures/2010pro4_numberprocedureage.pdf. Accessed January 23, 2014.

5. Dauty M, Schmitt X, Menu P, Rousseau B, Dubois C. Using the Risk Assessment and Predictor Tool (RAPT) for patients after total knee replacement surgery. Ann Phys Rehabil Med. 2012;55:4-15.

6. Ethgen O, Bruyere O, Richy F, Dardennes C, Reginster JY. Health-related quality of life in total hip and total knee arthroplasty. A qualitative and systematic review of the literature. J Bone Joint Surg Am. 2004;86:963-974.

7. Lavernia CJ, Guzman JF, Gachupin-Garcia A. Cost effectiveness and quality of life in knee arthroplasty. Clin Orthop Relat Res. 1997;345:134-139.

8. Losina E, Walensky RP, Kessler CL, Emrani PS, Reichmann WM, Wright EA, Holt HL, Solomon DH, Yelin E, Paltiel AD, Katz JN. Cost-effectiveness of total knee arthroplasty in the United States: patient risk and hospital volume. Arch Intern Med. 2009;169:1113-1121; discussion 1121-1122.

9. Malchau H, Garellick G, Eisler T, Karrholm J, Herberts P. Presidential guest address: the Swedish Hip Registry: increasing the sensitivity by patient outcome data. Clin Orthop Relat Res. 2005;441:19-29.

10. Maradit Kremers H, Visscher SL, Moriarty JP, Reinalda MS, Kremers WK, Naessens JM, Lewallen DG. Determinants of direct medical costs in primary and revision total knee arthroplasty. Clin Orthop Relat Res. 2013;471:206-214.

11. Oldmeadow LB, McBurney H, Robertson VJ. Predicting risk of extended inpatient rehabilitation after hip or knee arthroplasty. $J$ Arthroplasty. 2003;18:775-779.

12. Rolfson O, Strom O, Karrholm J, Malchau H, Garellick G. Costs related to hip disease in patients eligible for total hip arthroplasty. J Arthroplasty. 2012;27:1261-1266.

13. SooHoo NF, Lieberman JR, Ko CY, Zingmond DS. Factors predicting complication rates following total knee replacement. $J$ Bone Joint Surg Am. 2006;88:480-485.

14. Tan C, Loo G, Pua YH, Chong HC, Yeo W, Ong PH, Lo NN, Allison G. Predicting discharge outcomes after total knee replacement using the Risk Assessment and Predictor Tool. Physiotherapy. 2014;100:176-181.

15. Tomek IM, Sabel AL, Froimson MI, Muschler G, Jevsevar DS, Koenig KM, Lewallen DG, Naessens JM, Savitz LA, Westrich JL, Weeks WB, Weinstein JN. A collaborative of leading health systems finds wide variations in total knee replacement delivery and takes steps to improve value. Health Aff (Millwood). 2012;31:1329-1338. 